\title{
$S$-asymptotically periodic solutions for an epidemic model with superlinear perturbation
}

\author{
Jing-Yun Zhao' ${ }^{1}$ Hui-Sheng Ding ${ }^{1 *}$ and Gaston M. N'Guérékata²
}

\author{
"Correspondence: \\ dinghs@mail.ustc.edu.cn \\ ${ }^{1}$ College of Mathematics and \\ Information Science, Jiangxi Normal \\ University, Nanchang, Jiangxi \\ 330022, People's Republic of China \\ Full list of author information is \\ available at the end of the article
}

\begin{abstract}
This paper is concerned with the existence of S-asymptotically periodic solutions for an epidemic model with superlinear perturbation. It seems that this is a first result as regards such a model with superlinear perturbation. We give sufficient conditions to ensure the existence of S-asymptotically periodic solutions for the problem addressed and give an example to show that our sufficient conditions can be satisfied.
\end{abstract}

MSC: 45G10; 34K14

Keywords: S-asymptotically periodic; delay integral equation; superlinear perturbation

\section{Introduction and preliminaries}

In [1], Cooke and Kaplan initiated the study of the following nonlinear delay integral equation:

$$
x(t)=\int_{t-\tau}^{t} f(s, x(s)) d s, \quad t \in \mathbb{R}
$$

which is a model for the spread of some infectious diseases. Since then, many mathematicians make an extensive study of the existence of periodic solutions and almost periodic solutions for equation (1.1) and its variants. We refer the reader to [2-13] and the references therein for some research work on this topic.

Especially, in 1997, Ait Dads and Ezzinbi considered the existence of positive almost periodic solutions for the following neutral integral equation:

$$
x(t)=\gamma x(t-\tau)+(1-\gamma) \int_{t-\tau}^{t} f(s, x(s)) d s, \quad t \in \mathbb{R},
$$

where $\gamma \in[0,1)$. Since the work of Ait Dads and Ezzinbi, several authors have made contributions on equation (1.2) and its variants (see, e.g., $[6,8]$ and the references therein). Especially, stimulated by the work of Ait Dads, Cieutat, and Lhachimi [5], the authors in [8] investigated the existence of positive pseudo almost periodic solution for the following 
more general neutral integral equation:

$$
x(t)=\alpha(t) x(t-\beta)+\int_{-\infty}^{t} a(t, t-s) f(s, x(s)) d s, \quad t \in \mathbb{R} .
$$

In fact, both equation (1.2) and equation (1.3) can be seen as a linear perturbation of equation (1.1). Then a natural question arises:

When are there bounded solutions for equation (1.1) with superlinear perturbations?

It seems that there is no literature about the existence of bounded solutions for equation (1.1) with superlinear perturbations until now. The aim of this paper is to give some answers to the above problem.

On the other hand, an interesting notion of $S$-asymptotically periodic functions was recently introduced and studied by several authors (see, e.g., [14] and the references therein). In fact, it turns out that $S$-asymptotically periodic functions are an important and interesting generalization of asymptotically periodic functions. It has attracted great interest from many authors studying $S$-asymptotically periodic functions and their applications in differential equations (especially abstract differential equations in Banach spaces). We refer the reader to [15-22] and the references therein for some recent contributions on this topic.

Stimulated by the work on equation (1.1) and the work on $S$-asymptotically periodic functions, in this paper, we will investigate the existence of $S$-asymptotically periodic solutions for the following delay integral equation with superlinear perturbations:

$$
x(t)=\alpha(t) x^{n}(t-\beta)+\int_{t-\tau(t)}^{t} f(s, x(s)) d s, \quad t \in \mathbb{R},
$$

where $n \geq 1$ and $\beta \geq 0$ are fixed constants, and $\alpha, \tau, f$ satisfy some conditions recalled in Section 2. Here, if we define

$$
(\mathfrak{O} x)(t)=\alpha(t) x^{n}(t-\beta)
$$

then $\mathfrak{O}(\lambda x)=\lambda^{n} \mathfrak{O} x$. In the case of $n=1, \mathfrak{O}$ is a linear operator. Thus, in this paper, we call $\mathfrak{O}$ superlinear in the case of $n>1$.

Throughout the rest of this paper, we denote by $\mathbb{N}$ the set of positive integers, by $\mathbb{R}$ the set of real numbers, and by $\mathbb{R}^{+}$the set of nonnegative real numbers.

Definition 1.1 A bounded and continuous function $f: \mathbb{R} \rightarrow \mathbb{R}$ is called $S$-asymptotically periodic if there exists $\omega>0$ such that $\lim _{t \rightarrow \infty}[f(t+\omega)-f(t)]=0$. We denote by $\operatorname{SAP}_{\omega}(\mathbb{R})$ the set of all such functions.

Remark 1.2 Note that our definition has a slight difference from [14], where a $S$ asymptotically periodic function is defined on $\mathbb{R}^{+}$.

Lemma 1.3 Let $f, g \in \operatorname{SAP}_{\omega}(\mathbb{R})$. Then the following assertions hold:

(a) $\operatorname{SAP}_{\omega}(\mathbb{R})$ is a Banach space under the supremum norm.

(b) $f(\cdot+s) \in \mathrm{SAP}_{\omega}(\mathbb{R})$ for every $s \in \mathbb{R}$.

(c) $f \cdot g \in \operatorname{SAP}_{\omega}(\mathbb{R})$. 
Proof One can prove (a) by using a very similar proof to that of [14], Proposition 3.5. Moreover, one can show (b) and (c) by directly using the definition of $\operatorname{SAP}_{\omega}(\mathbb{R})$. We omit the details here.

\section{Main results}

We first establish two lemmas about $S$-asymptotically periodic functions.

Lemma 2.1 Let $f: \mathbb{R} \times \mathbb{R}^{+} \rightarrow \mathbb{R}^{+}$be a continuous function satisfying $f(t, \lambda x) \geq \lambda f(t, x)$ for all $t \in \mathbb{R}$ and $x \in \mathbb{R}^{+}$. Moreover, for every $x>0, f(\cdot, x) \in \mathrm{SAP}_{\omega}(\mathbb{R})$ and $g \in \mathrm{SAP}_{\omega}(\mathbb{R})$ with $\inf _{t \in \mathbb{R}} g(t)>0$. Then $f(\cdot, g(\cdot)) \in \operatorname{SAP}_{\omega}(\mathbb{R})$.

Proof Let $a=\inf _{t \in \mathbb{R}} g(t)$ and $b=\sup _{t \in \mathbb{R}} g(t)$. Then $0<a \leq b<+\infty$. Noting that $f(t, \lambda x) \geq$ $\lambda f(t, x)$, by [5], Lemma 3.1, there exists $L>0$ such that

$$
|f(t, x)-f(t, y)| \leq L|x-y|, \quad t \in \mathbb{R}, x, y \in[a, b] .
$$

Then it is easy to see that $f(\cdot, g(\cdot))$ is bounded and continuous.

For every $n \in \mathbb{N}$ and $t \in \mathbb{R}$, there exists $i_{t} \in\{0,1,2, \ldots, n\}$ such that $\left|g(t)-a-\frac{i_{t}}{n}(b-a)\right| \leq$ $\frac{b-a}{n}$. Then, for every $n \in \mathbb{N}$ and $t \in \mathbb{R}$, we have

$$
\begin{aligned}
&|f(t+\omega, g(t+\omega))-f(t, g(t))| \\
& \leq|f(t+\omega, g(t+\omega))-f(t, g(t+\omega))|+|f(t, g(t+\omega))-f(t, g(t))| \\
& \leq|f(t+\omega, g(t+\omega))-f(t, g(t+\omega))|+L|g(t+\omega)-g(t)| \\
& \leq \frac{2 L(b-a)}{n}+\left|f\left(t+\omega, a+\frac{i_{t+\omega}}{n}(b-a)\right)-f\left(t, a+\frac{i_{t+\omega}}{n}(b-a)\right)\right| \\
& \quad+L|g(t+\omega)-g(t)| \\
& \leq \frac{2 L(b-a)}{n}+\sum_{i=0}^{n}\left|f\left(t+\omega, a+\frac{i}{n}(b-a)\right)-f\left(t, a+\frac{i}{n}(b-a)\right)\right| \\
& \quad+L|g(t+\omega)-g(t)|,
\end{aligned}
$$

combining this with

$$
\lim _{t \rightarrow \infty}\left|f\left(t+\omega, a+\frac{i}{n}(b-a)\right)-f\left(t, a+\frac{i}{n}(b-a)\right)\right|=0, \quad i=0,1,2, \ldots, n,
$$

we conclude that $\lim _{t \rightarrow \infty}|f(t+\omega, g(t+\omega))-f(t, g(t))|=0$, i.e., $f(\cdot, g(\cdot)) \in \operatorname{SAP}_{\omega}(\mathbb{R})$.

Lemma 2.2 Let $f, \tau \in \operatorname{SAP}_{\omega}(\mathbb{R})$. Then $F \in \operatorname{SAP}_{\omega}(\mathbb{R})$, where $F(t)=\int_{t-\tau(t)}^{t} f(s) d s$ for all $t \in \mathbb{R}$.

Proof Note that

$$
\begin{aligned}
|F(t+\omega)-F(t)| & =\left|\int_{t+\omega-\tau(t+\omega)}^{t+\omega} f(s) d s-\int_{t-\tau(t)}^{t} f(s) d s\right| \\
& =\left|\int_{t-\tau(t+\omega)}^{t} f(s+\omega) d s-\int_{t-\tau(t)}^{t} f(s) d s\right|
\end{aligned}
$$




$$
\begin{aligned}
& \leq\left|\int_{t-\tau(t+\omega)}^{t-\tau(t)} f(s+\omega) d s\right|+\left|\int_{t-\tau(t)}^{t}[f(s+\omega)-f(s)] d s\right| \\
& \leq\|f\| \cdot|\tau(t+\omega)-\tau(t)|+\|\tau\| \cdot \sup _{t-\|\tau\| \leq s \leq t+\|\tau\|}|f(s+\omega)-f(s)|,
\end{aligned}
$$

it follows from $f, \tau \in \operatorname{SAP}_{\omega}(\mathbb{R})$ that $\lim _{t \rightarrow \infty}[F(t+\omega)-F(t)]=0$. Also, it is not difficult to see that $F$ is bounded and continuous. This completes the proof.

For convenience, we list some assumptions.

(H0) $\alpha, \tau \in \operatorname{SAP}_{\omega}(\mathbb{R})$ are nonnegative.

(H1) $f: \mathbb{R} \times \mathbb{R}^{+} \rightarrow \mathbb{R}^{+}$is a continuous function satisfying that $f(t, \cdot)$ is nondecreasing in $\mathbb{R}^{+}$for every $t \in \mathbb{R}, f(\cdot, x) \in \operatorname{SAP}_{\omega}(\mathbb{R})$ for every $x \in \mathbb{R}^{+}$, and there exists $\phi:(0,1) \rightarrow(0,1]$ such that $f(t, \lambda x) \geq \phi(\lambda) f(t, x)$ for all $t \in \mathbb{R}, \lambda \in(0,1)$ and $x \in \mathbb{R}^{+}$.

(H2) There exist two constants $M>\varepsilon>0$ such that for all $t \in \mathbb{R}$,

$$
\alpha(t) \varepsilon^{n}+\int_{t-\tau(t)}^{t} f(s, \varepsilon) d s \geq \varepsilon \quad \text { and } \quad \alpha(t) M^{n}+\int_{t-\tau(t)}^{t} f(s, M) d s \leq M .
$$

(H3) For every $\lambda \in(0,1), \phi(\lambda)>\lambda+r_{0}\left(\lambda-\lambda^{n}\right)$, where

$$
r_{0}=\frac{\bar{\alpha} \cdot M^{n}}{\inf _{t \in \mathbb{R}} \int_{t-\tau(t)}^{t} f\left(s, \frac{\varepsilon^{2}}{M}\right) d s}<+\infty \quad \text { and } \quad \bar{\alpha}=\sup _{t \in \mathbb{R}} \alpha(t) .
$$

Theorem 2.3 Assume that (H0)-(H3) hold. Then equation (1.4) has a S-asymptotically periodic solution $x^{*}$ with $\inf _{t \in \mathbb{R}} x^{*}(t)>0$.

Proof Let

$$
\begin{aligned}
& E=\left\{x \in \operatorname{SAP}_{\omega}(\mathbb{R}): \inf _{t \in \mathbb{R}} x(t)>0\right\}, \\
& (B x)(t)=\int_{t-\tau(t)}^{t} f(s, x(s)) d s, \quad(C x)(t)=\alpha(t) x^{n}(t-\beta), \quad t \in \mathbb{R}, x \in E,
\end{aligned}
$$

and

$$
(A x)(t)=(B x)(t)+(C x)(t), \quad t \in \mathbb{R}, x \in E .
$$

Noting that $r_{0}=\frac{\bar{\alpha} \cdot M^{n}}{\inf _{t \in \mathbb{R}} \int_{t-\tau(t)}^{t} f\left(s, \frac{\varepsilon^{2}}{M}\right) d s}$, for every $x \in E$ with $\frac{\varepsilon^{2}}{M} \leq x(t) \leq M$ for all $t \in \mathbb{R}$, we have

$$
(C x)(t) \leq \bar{\alpha} \cdot M^{n}=r_{0} \inf _{t \in \mathbb{R}} \int_{t-\tau(t)}^{t} f\left(s, \frac{\varepsilon^{2}}{M}\right) d s \leq r_{0}(B x)(t), \quad t \in \mathbb{R} .
$$

Combining this with (H1), for all $t \in \mathbb{R}, \lambda \in(0,1)$, and $x \in E$ with $\frac{\varepsilon^{2}}{M} \leq x(t) \leq M$ for all $t \in \mathbb{R}$, we have

$$
\begin{aligned}
A(\lambda x)(t) & =B(\lambda x)(t)+C(\lambda x)(t) \geq \phi(\lambda)(B x)(t)+\lambda^{n}(C x)(t) \\
& =\lambda(A x)(t)+[\phi(\lambda)-\lambda](B x)(t)+\left[\lambda^{n}-\lambda\right](C x)(t)
\end{aligned}
$$




$$
\begin{aligned}
& \geq \lambda(A x)(t)+\left[\phi(\lambda)-\lambda-\left(\lambda-\lambda^{n}\right) r_{0}\right](B x)(t) \\
& \geq\left[\lambda+\frac{\phi(\lambda)-\lambda-\left(\lambda-\lambda^{n}\right) r_{0}}{1+r_{0}}\right](A x)(t) \\
& =\psi(\lambda)(A x)(t)
\end{aligned}
$$

where $\psi(\lambda)=\lambda+\frac{\phi(\lambda)-\lambda-\left(\lambda-\lambda^{n}\right) r_{0}}{1+r_{0}}>\lambda$ for every $\lambda \in(0,1)$ by (H3). Especially, it follows from (2.1) that, for all $t \in \mathbb{R}, \lambda \in(0,1]$ and $x \in E$ with $\frac{\varepsilon^{2}}{M} \leq x(t) \leq M$ for all $t \in \mathbb{R}$, we have

$$
A(\lambda x)(t) \geq \lambda A(x)(t)
$$

Let $u_{0}(t) \equiv \varepsilon, v_{0}(t) \equiv M$, and

$$
u_{k}(t)=\left(A u_{k-1}\right)(t), \quad v_{k}(t)=\left(A v_{k-1}\right)(t), \quad t \in \mathbb{R}, k=1,2, \ldots
$$

By (H2), we know that $u_{1}(t) \geq \varepsilon$ and $v_{1}(t) \leq M$ for every $t \in \mathbb{R}$. Then, by using the fact that $f(t, \cdot)$ is nonincreasing in $\mathbb{R}^{+}$for every $t \in \mathbb{R}$, we conclude that

$$
\varepsilon \leq u_{1}(t) \leq u_{2}(t) \leq \cdots \leq u_{k}(t) \leq \cdots \leq v_{k}(t) \leq \cdots \leq v_{2}(t) \leq v_{1}(t) \leq M, \quad t \in \mathbb{R}
$$

In addition, combining (H1) with Lemma 1.3, Lemma 2.1, and Lemma 2.2, we can conclude that for every $k \in \mathbb{N}, u_{k} \in \operatorname{SAP}_{\omega}(\mathbb{R})$ and $v_{k} \in \operatorname{SAP}_{\omega}(\mathbb{R})$.

Let $\mu_{k}=\sup \left\{\mu>0: u_{k}(t) \geq \mu v_{k}(t), t \in \mathbb{R}\right\}$. Then

$$
\frac{\varepsilon}{M} \leq \mu_{1} \leq \cdots \leq \mu_{k} \leq 1
$$

Set $\lim _{k \rightarrow \infty} \mu_{k}=\mu_{0}$. It is easy to see that $\mu_{0} \in\left[\frac{\varepsilon}{M}, 1\right]$. We claim that $\mu_{0}=1$. In fact, if $\mu_{0}<1$, noting that $\frac{\varepsilon^{2}}{M} \leq \mu_{0} v_{k}(t) \leq M$ for all $t \in \mathbb{R}$ and $k \in \mathbb{N}$, by (2.2) and (2.1), we have

$$
\begin{aligned}
u_{k+1}(t) & =\left(A u_{k}\right)(t) \\
& \geq A\left(\mu_{k} v_{k}\right)(t) \\
& =A\left(\frac{\mu_{k}}{\mu_{0}} \mu_{0} v_{k}\right)(t) \\
& \geq \frac{\mu_{k}}{\mu_{0}} A\left(\mu_{0} v_{k}\right)(t) \\
& \geq \frac{\mu_{k}}{\mu_{0}} \psi\left(\mu_{0}\right) v_{k+1}(t),
\end{aligned}
$$

which means that $\mu_{k+1} \geq \frac{\mu_{k}}{\mu_{0}} \psi\left(\mu_{0}\right)$, i.e.,

$$
\frac{\mu_{k+1}}{\mu_{k}} \geq \frac{\psi\left(\mu_{0}\right)}{\mu_{0}}>1 .
$$

Then we have

$$
\mu_{k+1} \geq \mu_{1}\left[\frac{\psi\left(\mu_{0}\right)}{\mu_{0}}\right]^{k} \rightarrow \infty
$$

and thus $\lim _{k \rightarrow \infty} \mu_{k}=\infty$. This is a contradiction. 
For every $k, m \in \mathbb{N}$ with $k>m$, we have

$$
0 \leq u_{k}(t)-u_{m}(t) \leq v_{k}(t)-u_{m}(t) \leq v_{m}(t)-u_{m}(t) \leq\left(1-\mu_{m}\right) v_{m}(t) \leq\left(1-\mu_{m}\right) M, \quad t \in \mathbb{R},
$$

which yields

$$
\sup _{t \in \mathbb{R}}\left|u_{k}(t)-u_{m}(t)\right| \leq\left(1-\mu_{m}\right) M \rightarrow 0, \quad m \rightarrow \infty
$$

Thus, there exists $x^{*} \in \operatorname{SAP}_{\omega}(\mathbb{R})$ such that $u_{k} \rightarrow x^{*}$ in $\operatorname{SAP}_{\omega}(\mathbb{R})$ as $k \rightarrow \infty$. It is easy to see that for all $t \in \mathbb{R}$,

$$
\varepsilon \leq u_{1}(t) \leq u_{2}(t) \leq \cdots \leq u_{k}(t) \leq \cdots \leq x^{*}(t) \leq \cdots \leq v_{k}(t) \leq \cdots \leq v_{2}(t) \leq v_{1}(t) \leq M .
$$

So we have

$$
0 \leq v_{k}(t)-x^{*}(t) \leq v_{k}(t)-u_{k}(t) \leq\left(1-\mu_{k}\right) M, \quad t \in \mathbb{R},
$$

which means that $v_{k} \rightarrow x^{*}$ in $\operatorname{SAP}_{\omega}(\mathbb{R})$ as $k \rightarrow \infty$. For all $k \in \mathbb{N}$ and $t \in \mathbb{R}$, since $u_{k}(t) \leq$ $x^{*}(t) \leq v_{k}(t)$, we have

$$
u_{k+1}(t)=\left(A u_{k}\right)(t) \leq\left(A x^{*}\right)(t) \leq\left(A v_{k}\right)(t)=v_{k+1}(t)
$$

Letting $k \rightarrow \infty$, we get $\left(A x^{*}\right)(t)=x^{*}(t)$ for all $t \in \mathbb{R}$, i.e.,

$$
x^{*}(t)=\alpha(t)\left[x^{*}(t-\beta)\right]^{n}+\int_{t-\tau(t)}^{t} f\left(s, x^{*}(s)\right) d s, \quad t \in \mathbb{R} .
$$

Thus $x^{*}$ is a $S$-asymptotically periodic solution of equation (1.4).

Next, we show that our assumptions can be satisfied by a simple example, which does not aim at generality.

Example 2.4 Let $n=\frac{3}{2}, \alpha(t) \equiv \frac{1}{20}, \beta=0, \tau(t) \equiv 1$, and

$$
f(t, x)=a(t) \sqrt{x}
$$

where $a \in \operatorname{SAP}_{\omega}(\mathbb{R})$ with $1 \leq \inf _{t \in \mathbb{R}} a(t) \leq \sup _{t \in \mathbb{R}} a(t) \leq \frac{13}{10}$.

Obviously, (H0) holds. It is easy to see that $(\mathrm{H} 1)$ holds with $\phi(\lambda)=\sqrt{\lambda}$. By a direct calculation, one can show that $(\mathrm{H} 2)$ holds with $\varepsilon=1$ and $M=2$. In addition, we have

$$
r_{0}=\frac{\bar{\alpha} \cdot M^{n}}{\inf _{t \in \mathbb{R}} \int_{t-\tau(t)}^{t} f\left(s, \frac{\varepsilon^{2}}{M}\right) d s} \leq \frac{1}{5}
$$

and for every $\lambda \in(0,1)$,

$$
\frac{\phi(\lambda)-\lambda}{\lambda-\lambda^{n}}=\frac{\sqrt{\lambda}-\lambda}{\lambda-\lambda^{\frac{3}{2}}}=\frac{1}{\sqrt{\lambda}}>1,
$$

which means that $(\mathrm{H} 3)$ holds. 
Remark 2.5 By using the approach in Theorem 2.3, one can also prove some similar results as regards the existence of almost periodic type solutions for equation (1.4).

\author{
Competing interests \\ None of the authors have any competing interests in the manuscript.
}

Authors' contributions

All authors contribute equally to this work.

\title{
Author details
}

${ }^{1}$ College of Mathematics and Information Science, Jiangxi Normal University, Nanchang, Jiangxi 330022, People's Republic of China. ${ }^{2}$ Department of Mathematics, Morgan State University, 1700 E. Cold Spring Lane, Baltimore, Maryland 21251, USA.

\section{Acknowledgements}

The work was partially supported by NSFC (11461034), the Program for Cultivating Young Scientist of Jiangxi Province (20133BCB23009), the NSF of Jiangxi Province (20143ACB21001), and the Foundation of Jiangxi Provincial Education Department (GJJ150342).

Received: 24 May 2016 Accepted: 23 August 2016 Published online: 30 August 2016

\section{References}

1. Cooke, KL, Kaplan, JL: A periodicity threshold theorem for epidemics and population growth. Math. Biosci. 31, 87-104 (1976)

2. Ait Dads, E, Ezzinbi, K: Almost periodic solution for some neutral nonlinear integral equation. Nonlinear Anal. 28, 1479-1489 (1997)

3. Ait Dads, E, Ezzinbi, K: Existence of positive pseudo-almost-periodic solution for some nonlinear infinite delay integral equations arising in epidemic problems. Nonlinear Anal. 41, 1-13 (2000)

4. Ait Dads, E, Cieutat, P, Lhachimi, L: Positive almost automorphic solutions for some nonlinear infinite delay integral equations. Dyn. Syst. Appl. 17, 515-538 (2008)

5. Ait Dads, E, Cieutat, P, Lhachimi, L: Positive pseudo almost periodic solutions for some nonlinear infinite delay integral equations. Math. Comput. Model. 49, 721-739 (2009)

6. Bellour, A, Ait Dads, E: Periodic solutions for nonlinear neutral delay integro-differential equations. Electron. J. Differ. Equ. 100, 9 (2015)

7. Ding, HS, N'Guérékata, GM: A note on the existence of positive bounded solutions for an epidemic model. Appl. Math. Lett. 26, 881-885 (2013)

8. Ding, HS, Chen, YY, N'Guérékata, GM: Existence of positive pseudo almost periodic solutions to a class of neutral integral equations. Nonlinear Anal. 74, 7356-7364 (2011)

9. Ezzinbi, K, Hachimi, MA: Existence of positive almost periodic solutions of functional equations via Hilbert's projective metric. Nonlinear Anal. 26, 1169-1176 (1996)

10. Fink, AM, Gatica, JA: Positive almost periodic solutions of some delay integral equations. J. Differ. Equ. 83, 166-178 (1990)

11. Long, W: Existence of positive almost automorphic solutions to a class of integral equations. Afr. Diaspora J. Math. 12, 48-56 (2011)

12. Torrejón, R: Positive almost periodic solutions of a state-dependent delay nonlinear integral equation. Nonlinear Anal. 20, 1383-1416 (1993)

13. Williams, LR, Leggett, RW: Nonzero solutions of nonlinear integral equations modeling infectious disease. SIAM J. Math. Anal. 13, 112-121 (1982)

14. Henríquez, HR, Pierri, M, Táboas, P: On S-asymptotically $\omega$-periodic functions on Banach spaces and applications. J. Math. Anal. Appl. 343, 1119-1130 (2008)

15. de Andrade, B, Cuevas, C: S-asymptotically $\omega$-periodic and asymptotically $\omega$-periodic solutions to semilinear Cauchy problems with nondense domain. Nonlinear Anal. 72, 3190-3208 (2010)

16. Cuevas, C, Lizama, C: S-asymptotically $\omega$-periodic solutions for semilinear Volterra equations. Math. Methods Appl. Sci. 33, 1628-1636 (2010)

17. Cuevas, C, deSouza, JC: S-asymptotically $\omega$-periodic solutions of semilinear fractional integro-differential equations. Appl. Math. Lett. 22, 865-870 (2009)

18. Dos Santos, JPC, Henríquez, HR: Existence of S-asymptotically $\omega$-periodic solutions to abstract integro-differential equations. Appl. Math. Comput. 256, 109-118 (2015)

19. Henríquez, HR, Pierri, M, Rolnik, V: Pseudo S-asymptotically periodic solutions of second-order abstract Cauchy problems. Appl. Math. Comput. 274, 590-603 (2016)

20. Pierri, M, Rolnik, V: On pseudo S-asymptotically periodic functions. Bull. Aust. Math. Soc. 87, 238-254 (2013)

21. Dimbour, W, N'Guérékata, GM: S-asymptotically $\omega$-periodic solutions to some classes of partial evolution equations. Appl. Math. Comput. 218, 7622-7628 (2012)

22. Dimbour, W, Mophou, G, N'Guérékata, GM: S-asymptotically periodic solutions for partial differential equations with finite delay. Electron. J. Differ. Equ. 2011(117), 12 (2011) 